\title{
The Coupling Impedance of the RHIC Injection Kicker System
}

\author{
H. Hahn \\ Brookhaven National Laboratory, Upton, NY 11973
}

\begin{abstract}
In this paper, results from impedance measurements on the RHIC injection kickers are reported. The kicker is configured as a " $\mathrm{C}$ " cross section magnet with interleaved ferrite and high-permittivity dielectric sections to achieve a travelling wave structure. The impedance was measured using the wire method in which a resistive match provides a smooth transition from the network analyzer to the reference line in the set-up. Accurate results are obtained by interpreting the forward scattering coefficient via the log-formula. The four kickers with their ceramic beam tubes contribute a $Z / n=0.22 \Omega /$ ring in the interesting frequency range from 0.1 to $1 \mathrm{GHz}$, and less above. At frequencies above $\sim 100 \mathrm{MHz}$, the impedance is ferrite dominated and not affected by the kicker terminations. Below $100 \mathrm{MHz}$, the Blumlein pulser with the $\sim 75$ $\mathrm{m}$ feeding cables is visible in the impedance but makes no significant contribution to the results. The measurements show that the kicker coupling impedance is tolerable without the need for impedance reducing measures.
\end{abstract}

\section{INTRODUCTION}

The RHIC injection kicker ${ }^{1}$ is configured as " $\mathrm{C}$ " cross section magnet with interspersed ferrite ${ }^{2}$ and dielectric ${ }^{3}$ blocks as shown in Fig. 1 The deflection properties of the kicker are dominated by the magnetic field and thus by the ferrite properties and its geometrical configuration. The dielectric blocks provide the capacitance required to achieve a transmission line kicker and to reduce the charcteristic impedance so as to match the $25 \Omega$ of the Blumlein pulser.

The longitudinal coupling impedance of a half-size kicker model was previously measured by Mane et al. ${ }^{4}$ with the results raising some concern due to the presence of sharp resonances in the $\mathrm{GHz}$ region. A more extensive study identified the origin of these resonances as local resonances in the dielectric blocks exposed to the beam. ${ }^{5}$ In the modified kicker configuration, the resonances were completely suppressed by removing the dielectric blocks at the sides and replacing them by ferrite. The two kicker versions are shown in Fig. 1.

1) Work performed under the auspices of the U. S. Department of Energy 


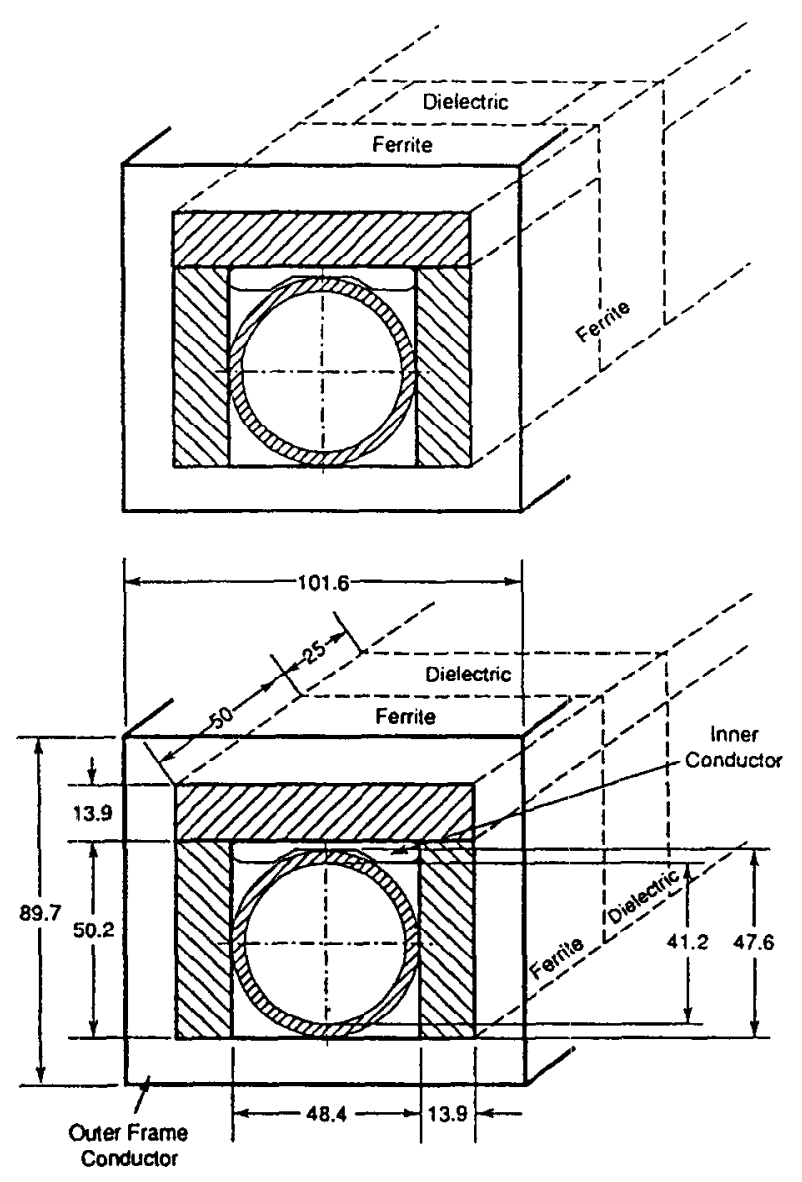

FIGURE 1. Geometry of original (bottom) and modified (top) RHIC injection kicker design.

In this paper, measured results for the longitudinal coupling impedance of a halfsize injection kicker model, both free-standing as well as part of the kicker system with Blumlein pulser, are presented.

\section{WIRE MEASUREMENT OF THE COUPLING IMPEDANCE}

The coupling impedance of the kicker system was measured with the existing bench setup for wire measurements previously used and described, ${ }^{4}$ but now using the wire method in which a resistive match provides a smooth transition from the $50 \Omega$ of the network analyzer to the characteristic impedance of the reference line in the setup. ${ }^{6}$ The advantage of this method stems from the simplicity of its calibration procedure and the fact that a sufficient approximation of the results for the impedance in terms of real/imaginary or amplitude/phase is directly provided by the network analyzer and can be plotted without post processing. However the 
results quoted were obtained using the more accurate "log-formula" appropriate for distributed systems.

The measurements were performed with the Hewlett-Packard Network Analyzer $8753 \mathrm{C}$ connected to the S-Parameter Test Set 85078A. The characteristic impedance of the connecting reference waveguides was measured directly to be $Z_{\text {ref }}=165 \Omega$ by shorting the output port of a waveguide section, for which the input impedance becomes

$$
Z_{\text {in }}=Z_{r e f} \tan \left(\frac{\pi}{2} \frac{f}{f_{\lambda / 4}}\right)
$$

Matching the $50 \Omega$ of the network analyzer to the $165 \Omega$ of the reference line is achieved by adding at the input port, a parallel resistor of $\sim 59.9 \Omega$ and a series resistor of $137.7 \Omega$ which provides forward and backward matching. At the output port, a series resistor of $\sim 115 \Omega$ provides forward matching. The frequency dependence of the carbon resistors and stray inductances/capapcitances destroys the match at higher frequencies, but is corrected by the calibration procedure.

The coupling impedance is obtained from the change in the forward scattering coefficient $S_{21 \text { DUT }}$ with respect to the reference system with $S_{21 \text { ref }}=1$ if properly calibrated. In the case of a single lumped disturbance, the coupling impedance is obtained by the well known formula

$$
Z_{h p}=2 Z_{\text {ref }} \frac{\left(1-S_{21 D U T} / S_{21 r e f}\right)}{\left(S_{21 D U T} / S_{21 r e f}\right)}
$$

which as a first approximation can also be used in the case of a distributed impedance such as the kicker. ${ }^{7}$ This formula is used by the $h p$ network analyzer and is quite useful in an exploratory study involving distributed impedances. However, if accurate results are required, at least the "log-formula"

$$
Z_{\log }=-2 Z_{r e f} \ln \frac{S_{21 D v T}}{S_{21 r e f}}
$$

in which the scattering coefficients are complex quantities should be used; ${ }^{8,9}$ although itself an approximation, it is extremely simple to use and sufficient in the present application.

\section{THE KICKER SYSTEM WITH CERAMIC BEAM TUBE}

The RHIC injection kicker system consists of four separate units, each $1.12 \mathrm{~m}$ long with separate pulser. The impedance measurements were performed in a halflength model with pulser and then extrapolated to the total system. ${ }^{10}$ The kicker will operate in air and thus requires a ceramic beam tube, the dimensions of which are $47.6 \mathrm{~mm}$ o.d. and $41.3 \mathrm{~mm}$ i.d. Insertion of the ceramic beam tube into the 

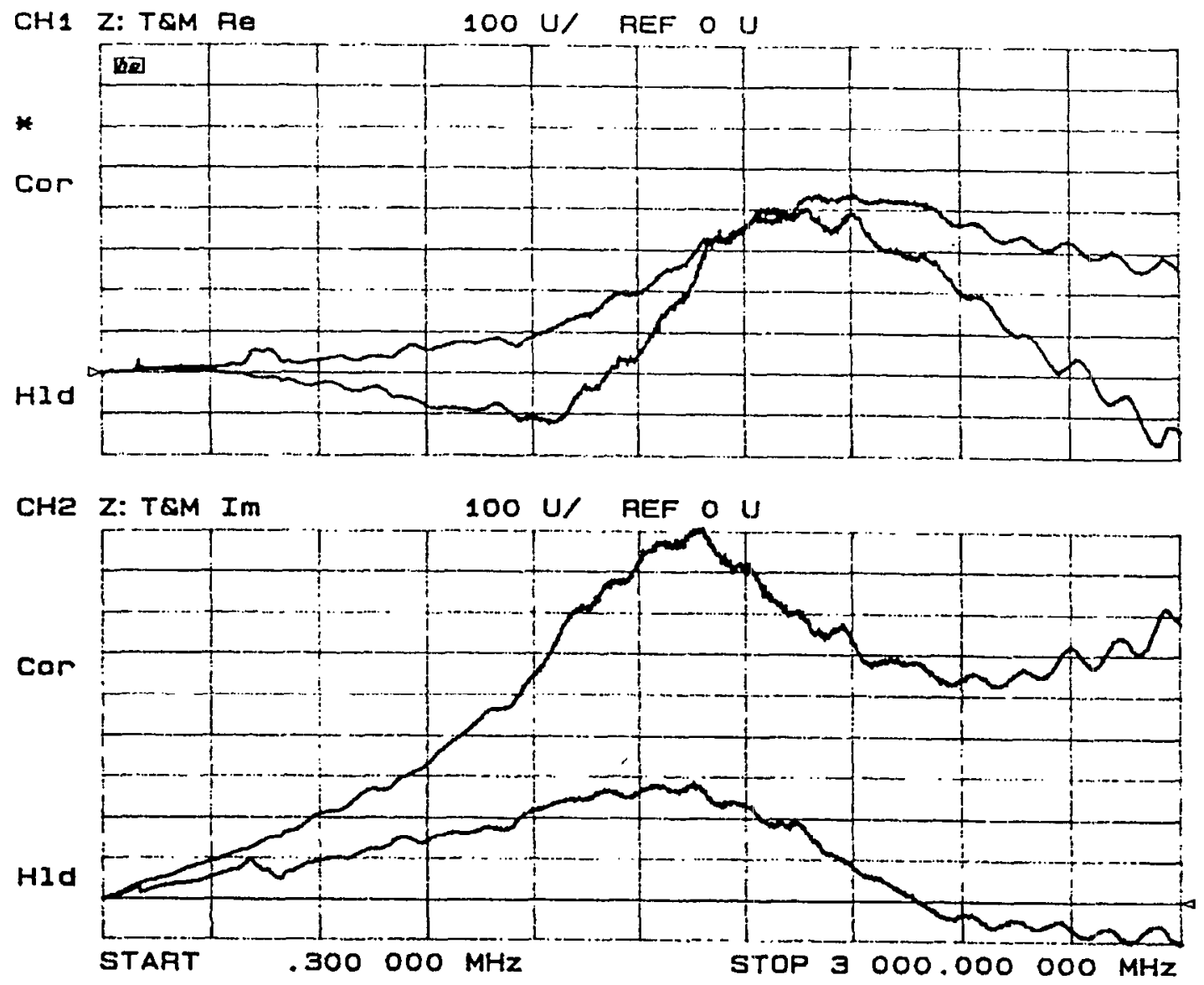

FIGURE 2. Comparison of impedance of kicker with and without beam tube.

kicker increases the capacity by $\sim 2 \%$ and thus the impedance by only $1 \%$. The coupling impedance of the kicker system, that is with the output port terminated and the input port connected to the pulser, has been measured with and without the ceramic beam tube. The hp impedance for both cases is compared in Fig. 2. Inspection of Fig. 2 shows an unphysical negative real part of the impedance. It has been verified, that this problem is caused by the use of the hp formula and can be corrected via the log-formula.

The results for the kicker system impedance are at frequencies above $\sim 100 \mathrm{MHz}$ independent of the port terminations and can be summarized by the impedance values with beam tube of

$$
|Z / n|=0.22 \Omega / \text { ring }
$$

and without beam tube of $|Z / n|=0.14 \Omega /$ ring, in the range up to $\sim 1 \mathrm{GHz}$, but lower at higher frequencies. Note that the impedance is resonance free in the measured region up to $3 \mathrm{GHz}$. The low-frequency end is discussed in the next section. 
Overall, one finds that the impedance contribution by the injection kicker is tolerable without the need for special impedance reduction measures such as a lowimpedance ( $R$-square, $R_{s q} \sim 1-10 \Omega$ ) coating. A low-impedance coating can limit the rise time and cause arcing at higher fields. A high-resistance coating $\left(R_{s q} \sim 1 \mathrm{M}\right.$ $\Omega$ ) will not reduce the coupling impedance but is required to prevent electrostatic charging of the beam tube, and will be provided in the RHIC kicker.

\section{LOW FREQUENCY COUPLING IMPEDANCE}

At frequencies bclow $\sim 100 \mathrm{MHz}$ the ferrites have low losses and the kicker acts as a transmission line magnetically coupled to the beam. As a result, the kicker terminations and in particular the pulser with the $\sim 75 \mathrm{~m}$ long connecting cable become visible in the coupling impedance, as seen in Fig. 3.
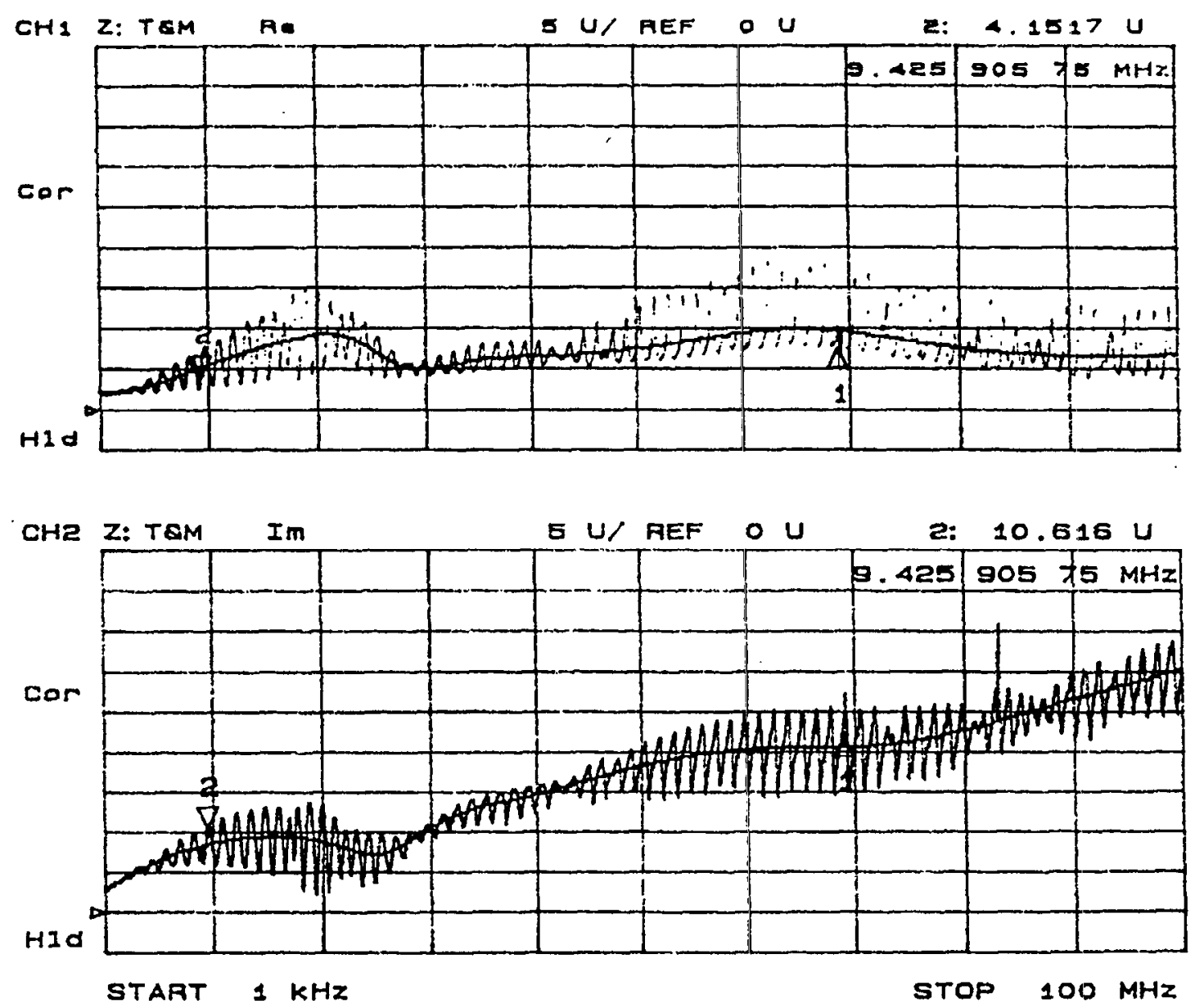

FIGURE 3. Low frequency coupling impedance of kicker system (rapid oscillation) and free standing kicker (smooth curve). 
Nassibian and Sacherer ${ }^{11}$ have theoretically analyzed this situation, but their results were limited to fully terminated kickers and a later report by Nassibian ${ }^{12}$ gave only expressions for the real part of the impedance. A generalized derivation of the longitudinal coupling impedance valid at "low" frequencies, is presented in the appendix. ${ }^{13}$ The kicker is still treated as a transformer-coupled uniform transmission line, but the constraints on the terminations are removed. The kicker is defined by its characteristic impedance $Z_{K}$ and the wave number $k_{K}$, both beamindependent parameters measurable directly on the magnet. The generalized theory of the coupling impedance has two additional free parameters: (1) the mutual inductance $M$ and (2) the "self"-inductance of the beam $L_{B}$. The values of these parameters are obtained from "wire"-measurements, using the theoretical expressions for their interpretation. As intuitively expected, the "self"-inductance of the beam follows from the results for the kicker open at both ports. The mutual inductance is expected to be

$$
M=\frac{1}{2} L_{K}(1+x / a)
$$

with $L_{K}$ the kicker inductance, $a$ the kicker half-aperture and $x$ the displacement from the center towards the bus bar. For a centered beam, one has $M=L_{K} / 2$.

The general expression for the coupling impedance is best handled via the computer program MACSYMA. The case of one port terminated, $R_{o}=Z_{K}$, and the input port represented by the general impedance $R_{i}=R+j X$ is of practical importance, as it reflects the typical situation of the kicker system. Here one finds

$$
\begin{aligned}
Z & =\frac{1}{2} Z_{K}\left(\frac{M}{L_{K}}\right)^{2}\left\{Z_{K}^{2}(1-\cos 2 \theta)\right. \\
& +4 Z_{K} R(1-\cos \theta) \\
& +\left(R^{2}+X^{2}\right)(3-4 \cos \theta+\cos 2 \theta) \\
& \left.-2 Z_{K} X(2 \sin \theta-\sin 2 \theta)\right\} / D \\
+ & Z_{K} \frac{L_{B}}{L_{K}} \theta-j \frac{1}{2} Z_{K}\left(\frac{M}{L_{K}}\right)^{2}\left\{Z_{K}^{2}(2 \theta-\sin 2 \theta)\right. \\
& +4 Z_{K} R(\theta-\sin \theta) \\
& +\left(R^{2}+X^{2}\right)(2 \theta-4 \sin \theta+\sin 2 \theta) \\
& -2 Z_{K} X(1-2 \cos \theta+\cos 2 \theta\} / D
\end{aligned}
$$

with the denominator $D=\left(Z_{K}+R\right)^{2}+X^{2}$,

$$
Z_{K}=\sqrt{\langle L>/<C>}
$$


and $\theta=\omega l \sqrt{(<L><\bar{C}>)}=2 \pi f / f_{2 \pi}$ the electrical length of the kickier where $\langle L\rangle,\langle C\rangle$ are the quantitites per unit length. Theory and measured results for the coupling impedance are in reasonable agreement as long as the "electrical length" of the kicker is less than one free-space wavelength. At higher frequencies, the assumption of magnetic coupling is still valid, but the kicker is no longer the ideal lossless, uniform transmission line and a more general treatment, for example based on equivalent circuits, is required. ${ }^{14}$

\section{REFERENCES}

1. H. Hahn, N. Tsoupas, and J. E. Tuozzolo, Proc. 1997 PAC, Vancouver, BC, Canada, p. 213.

2. CMD-5005 by Ceramic Magnetics, Inc., Fairfield, NJ

3. MCT-100 by Trans-Tech, Inc., Adamstown, MD.

4. V. Mane, S. Peggs. D. Trbojevic, and W. Zhang, Proc. 1995 PAC, Dallas, TX, p. 3134.

5. H. Hahn, M. Morvillo, A. Ratti, BNL Report AD/RHIC/RD-95 (1995).

6. A. Ratti, Fourth EPAC, London 1994, vol. 2, p. 1262.

7. H. Hahn and F. Pedersen, Report BNL \#50870 (1978).

8. L. S. Walling, D. E. McMurry, D. V. Neuffer, and H. A. Thiessen, Nucl. Instr. Meth., A 281, p. 433 (1989).

9. V. G. Vaccaro, et al, Proc. 1993 PAC, Washington, D.C., p. 2154 (1993).

10. H. Hahn and A. Ratti, BNL Report AD/RHIC/RD-105 (1996).

11. G. Nassibian and F. Sacherer, Nucl. Instr. \& Meth., vol. 159, p. 21 (1979).

12. G. Nassibian, Report CERN/PS 84-25(BR) (1984) and CERN/PS 85-68(BR) (1986).

13. H. Hahn and A. Ratti, BNL Report AD/RHIC/RD-111 (1997).

14. H. Hahn and A. Ratti, Proc. 1997 PAC, Vancouver, BC, Canada, p.216; BNL Report $\mathrm{AD} / \mathrm{RHIC} / \mathrm{RD}-112$ (1997). 


\section{APPENDIX: Theoretical Low-Frequency Coupling Impedance of Transmission Line Kickers}

In contrast to the "good" agreement between experiment and theory reported by Nassibian, the results from the RHIC injection kicker impedance measurements showed significant differences with the above theories. Furthermore, the impedance measurements of the kicker system can only be done with one port resistor-terminated and the other changing with frequency from short to open due to the long feeding cables. The need to amend the intrinsic limitations of the Sacherer-Nassibian theories thus provided the impetus for the present study.

In the low-frequency range here considered, the kicker acts as a transmission line with uniform, albeit anisotropic properties. The kicker and the beam are treated as magnetically coupled transmission lines, for which the differential equations are well known. By limiting the considerations to the extreme relativistic case, where the space charge effect can be neglected, the beam can be represented by a "transmission line" in which the inductance per unit length, $L_{B} / l$, is determined by the coupling impedance of the un-terminated kicker, and the capacitance is negligible. One finds, with the harmonic time dependence $\exp (j \omega t)$ suppressed, the following set of differential equations in the position-dependent variables $i_{\mathrm{K}}, u_{\mathrm{K}}, u_{\mathrm{B}}$ representing the kicker current, kicker voltage, and beam voltage respectively

$$
\begin{aligned}
\frac{\mathrm{d} u_{\mathrm{K}}}{\mathrm{d} s} & =-j k_{\mathrm{K}} Z_{\mathrm{K}} i_{\mathrm{K}}+j \frac{M}{L_{\mathrm{K}}} k_{\mathrm{K}} Z_{\mathrm{K}} i_{\mathrm{B}} \\
\frac{\mathrm{d} i_{\mathrm{K}}}{\mathrm{d} s} & =-j k_{\mathrm{K}} u_{\mathrm{K}} / Z_{\mathrm{K}} \\
\frac{\mathrm{d} u_{\mathrm{B}}}{\mathrm{d} s} & =j \frac{M}{L_{\mathrm{K}}} k_{\mathrm{K}} Z_{\mathrm{K}} i_{\mathrm{K}}-j \frac{L_{\mathrm{B}}}{L_{\mathrm{K}}} k_{\mathrm{K}} Z_{\mathrm{K}} i_{\mathrm{B}}
\end{aligned}
$$

Assuming an extreme relativistic, filamentary beam current of unit strength

$$
i_{\mathrm{B}}=e^{-j k s}
$$

one obtains the coupling impedance from

$$
Z=-\int_{0}^{l} \frac{\mathrm{d} u_{\mathrm{B}}}{\mathrm{d} s} e^{j k s} \mathrm{~d} s
$$

were $k=\omega / c, k_{\mathrm{K}}=\omega \sqrt{\langle L\rangle\langle C\rangle}$ and $Z_{\mathrm{K}}=\sqrt{\langle L\rangle /\langle C\rangle}$.

The solution of the above differential equations are found without difficulty, for example by means of the MACSYMA program, together with the boundary conditions established by the kicker input and output terminations, $R_{\mathrm{i}}$ and $R_{\mathrm{o}}$ 


$$
\begin{aligned}
& u_{\mathrm{K}}(s=0)=-R_{i} i_{\mathrm{K}}(s=0) \\
& u_{\mathrm{K}}(s=l)=R_{o} i_{\mathrm{K}}(s=l)
\end{aligned}
$$

The general expression for the coupling impedance is somewhat lengthy, but reduces at low frequencies, where $k$ \& $k_{\mathrm{K}}$, to a manageable size. Several special cases must be expected in the field or are accessible to measurement and thus are of theoretical interest. The case of one port terminated with the characteristic impedance and the other with a general impedance representing the pulser and connecting cables is given in the main part of this paper. A few simpler cases relevant to bench measurements are discussed here:

1. Case of $R_{\mathrm{i}}=\infty$ and $R_{\mathrm{o}}=\infty$

$$
\begin{aligned}
Z= & j Z_{\mathrm{K}} \frac{L_{\mathrm{B}}}{L_{\mathrm{K}}}\left(1-\kappa^{2}\right) \theta \\
& +j 2 Z_{\mathrm{K}}\left(\frac{M}{L_{\mathrm{K}}}\right)^{2} \frac{1-\cos \theta}{\sin \theta}
\end{aligned}
$$

with the magnetic coupling coefficient

$$
\kappa^{2}=\frac{M^{2}}{L_{\mathrm{K}} L_{\mathrm{B}}}
$$

This case, i.e. with both kicker ports open, clearly shows the inadequacy of the original Nassibian-Sacherer theory which predicts a singularity in the limit of $\theta \rightarrow 0$, in contrast to the experimental and the present theoretical result

$$
\lim _{\theta \rightarrow 0} Z=j Z_{\mathrm{K}} \frac{L_{\mathrm{B}}}{L_{\mathrm{K}}} \theta=j \omega L_{\mathrm{B}}
$$

2. Case of $R_{\mathrm{i}}=Z_{\mathrm{K}}$ and $R_{\mathrm{o}}=Z_{\mathrm{K}}$

$$
\begin{aligned}
Z= & Z_{\mathrm{K}}\left(\frac{M}{L_{\mathrm{K}}}\right)^{2}(1-\cos \theta) \\
& +j Z_{\mathrm{K}} \frac{L_{\mathrm{B}}}{L_{\mathrm{K}}}\left\{\left(1-\kappa^{2}\right) \theta+\kappa^{2} \sin \theta\right\}
\end{aligned}
$$


It is to be noted, that the above expressions for the real part of the coupling impedance is identical to Nassibian's result, whereas the imaginary part differs. At very low frequencies and in the limit of $\theta \gg 1$ one finds

$$
\begin{aligned}
& \lim _{\theta \rightarrow 0} Z=j Z_{K} \frac{L_{\mathrm{B}}}{L_{\mathrm{K}}} \theta=j \omega L_{\mathrm{B}} \\
& \lim _{\theta \rightarrow \infty} Z=j Z_{\mathrm{K}} \frac{L_{\mathrm{B}}}{L_{\mathrm{K}}}\left(1-\kappa^{2}\right) \theta=j \omega L_{\mathrm{B}}\left(1-\kappa^{2}\right)
\end{aligned}
$$

comparing these limits with wire- measurements yields $L_{\mathrm{B}}$ and $\kappa$ since the parameters $Z_{\mathrm{K}}$ and $L_{\mathrm{K}}$ are known from previous kicker measurements. The smooth curve in Fig 3 represents this case with input and output fully matched. The results can be fitted with $L_{\mathrm{B}}=132 \mathrm{nH}$ and $\left(1-\kappa^{2}\right)=0.325$, leading to

$$
L_{\mathrm{B}} / L_{\mathrm{K}}=0.18, M / L_{\mathrm{K}}=0.34 \text {, and } \kappa=0.82 .
$$

The values thus obtained can then be checked by comparison with the following two cases.

3. Case of $R_{i}=Z_{\mathrm{K}}$ and $R_{0}=0$

$$
\begin{aligned}
Z= & \frac{1}{2} Z_{\mathrm{K}}\left(\frac{M}{L_{\mathrm{K}}}\right)^{2}(1-\cos 2 \theta) \\
& +j Z_{\mathrm{K}} \frac{L_{\mathrm{B}}}{L_{\mathrm{K}}}\left\{\left(1-\kappa^{2}\right) \theta+\frac{1}{2} \kappa^{2} \sin 2 \theta\right\}
\end{aligned}
$$

4. Case of $R_{\mathrm{i}}=Z_{\mathrm{K}}$ and $R_{\mathrm{o}}=\infty$

$$
\begin{aligned}
Z= & \frac{1}{2} Z_{\mathrm{K}}\left(\frac{M}{L_{\mathrm{K}}}\right)^{2}(3-4 \cos \theta+\cos 2 \theta) \\
& +j Z_{\mathrm{K}} \frac{L_{\mathrm{B}}}{L_{\mathrm{K}}}\left\{\left(1-\kappa^{2}\right) \theta+2 \kappa^{2} \sin \theta-\frac{1}{2} \kappa^{2} \sin 2 \theta\right\}
\end{aligned}
$$

Theory and measured results for the coupling impedance are in reasonable agreement at "low" frequencies. At higher frequencies, the assumption of magnetic coupling is still valid, but the kicker is no longer the ideal lossless, uniform transmission line and a more general treatment, for example based on equivalent circuits, is required. 